\title{
Estudio aerodinámico del avión de entrenamiento de vuelo "KUNTUR"
}

\section{Aerodynamic study of the plane of training flight "KUNTUR"}

\author{
Jhonatan Paín ${ }^{1}$, Gabriel Cárdenas ${ }^{1}$, José Luna ${ }^{1,2}$ y Luis Romero ${ }^{1,3}$ \\ ${ }^{1}$ Escuela de Oficiales de la Fuerza Aérea del Perú. Av. Jorge Chávez S/N, Surco \\ ${ }^{2}$ Facultad de Ingeniería Ambiental, Universidad Alas Peruanas. Av. P. Ruíz Gallo 251, Pueblo Libre. \\ ${ }^{3}$ Facultad de Ingeniería, Universidad Privada Norbert Wiener. Jr. Larrabure y Unanue 110, Lima.
}

\section{RESUMEN}

El presente trabajo se realizó en las instalaciones de la Escuela de Oficiales y del Servicio de Mantenimiento de la Fuerza Aérea del Perú, ubicadas en la Base Aérea "Las Palmas" - Surco, con el objetivo de determinar la respuesta aerodinámica del avión de entrenamiento BD-4BP, conocido como "Kuntur", a la velocidad de crucero de 174 MPH $[1,2]$. El Kuntur, es un avión diseñado y construido en el Perú y cuenta con tecnología hibrida, es por ello la importancia de desarrollar este trabajo.

Para el desarrollo de la presente investigación se aplicó la metodología deductiva, con lo cual se pudieron determinar los vectores de velocidad y presión en torno al avión, para ello se empleó el software computacional en dinámica de fluidos CFD "Fluent", el cual realiza un cálculo basado en el método de volúmenes finitos [3,4].

Los materiales que se utilizaron fueron, una computadora personal Pentium IV con alta velocidad de procesamiento de datos, el software CDF Fluent v6.2, modelos a escala y un túnel de viento subsónico. La simulación es realizada en 2D y primeramente se malla el avión, para lo cual se definen las estructuras y posteriormente se colocan los parámetros aerodinámicos (velocidad de avión, presión atmosférica, viscosidad del fluido, número de Reynolds, tipo de flujo, cantidad de interacciones), obteniéndose con ello valores que luego de realizar el tratamiento estadístico son presentados en tablas y gráficas.

Los resultados presentados en este trabajo corresponden a la simulación y muestran los perfiles de velocidad y de presión en los diferentes puntos de la aeronave; así también, las evaluaciones son realizadas a diferentes ángulos de ataque de la aeronave observándose la turbulencia en torno al perfil, concluyéndose que el ángulo de ataque no sobrepase los $18^{\circ}$ porque entra en perdida. Se recomienda continuar con la investigación en 3 dimensiones, para que complemente el diseño y performance del avión de entrenamiento Kuntur.

Descriptores: Aerodinámica, Kuntur, velocidad de crucero, simulación, CFD Fluent, volúmenes finitos.

\section{ABSTRACT}

The present work realized in the facilities of the School of Officials and of the Service of Maintenance of the Air Force of Peru, located in the Air Base "Las Palmas" - Rut, with the aim to determine the aerodynamic response of the plane of training BD-4BP, known as "Kuntur", to the cruising speed of $174 \mathrm{MPH}[1,2]$. The Kuntur, it is a plane designed and constructed in Peru and possesses hybrid technology, there is for it the importance of developing this work.

For the development of the present investigation the deductive methodology was applied, with which they could determine the vectors of speed and pressure concerning the plane, for it there used the computational software in dynamics of fluids - CFD "Fluent", which realizes a calculation based on the method of finite volumes [3,4].

Materials that were in use were, a personal computer Pentium IV with high speed of processing of information, the software CDF Fluent v6.2, models to scale and a tunnel of subsonic wind. The simulation is realized in 2D and first mesh the plane, for which the structures are defined and later there are placed the aerodynamic parameters (speed of plane, atmospheric pressure, viscosity of the fluid, number of Reynolds, type of flow, quantity of interactions), there being obtained by it values that after realizing the statistical treatment are presented in tables and graphs.

The results presented in this work correspond to the simulation and show the profiles of speed and of pressure in the different points of the aircraft; this way also, the evaluations are realized to different angles of assault of the aircraft the turbulence being observed concerning the profile, concluding that the angle of assault does not exceed the $18^{\circ}$ that it enters loss. It is recommended to continue by the investigation in 3 dimensions, in order that it complements the design and performance of the plane of training Kuntur.

Keywords: Aerodynamics, Kuntur, cruising speed, simulation, CFD Fluent, finite volumes 


\section{INTRODUCCIÓN}

La Escuela de Oficiales de la Fuerza Aérea del Perú es una institución de formación académica y militar; dentro de las actividades académicas se encuentra la investigación, por dicho motivo, se están realizando proyectos para la generación de artículos científicos y tesis. Por dicho motivo se realizan investigaciones en los campos aplicativos como son la Administración Aeroespacial, Aeronáutica, Electrónica y Sistemas, en tal sentido se ha aprobado la investigación en el campo de la Aeronáutica con el avión de entrenamiento BD4BP el cual se le conoce como KUNTUR. El Kuntur es una aeronave de tipo monoplano de construcción mixta (aluminio y material compuesto), ligera, experimental de categoría normal, para entrenamiento básico diurno y nocturno en condiciones meteorológicas visuales de alta performance y moderno. El mismo que ha sido diseñado para cumplir o superar los requisitos estructurales establecidos para la producción de aviones según lo especificado en las especificaciones FAP $[1,2]$. La presente investigación se justifica por que esta aeronave ha sido diseñada y construida en los talleres del Servicio de mantenimiento de la FAP - SEMAN y por poseer un perfil único, hace necesario que se estudie su aerodinámica para determinar como son las fuerzas de sustentación y arrastre; es por ello que el objetivo es la de determinar la respuesta aerodinámica del avión de entrenamiento BD-4BP. Para la presente investigación se asumirá que se está bajo los siguientes supuestos:

$\begin{array}{lll}\text { Presión } & : & 1 \mathrm{~atm} \\ \text { Altura } & : & 0 \mathrm{msnm} \\ \text { Temperatura } & : & 20^{\circ} \mathrm{C} \\ \text { Densidad del aire } & : & 1,225 \mathrm{~kg} / \mathrm{m} 3 \\ \text { Viscosidad del aire } & : & 1,79 \times 10-5\end{array}$

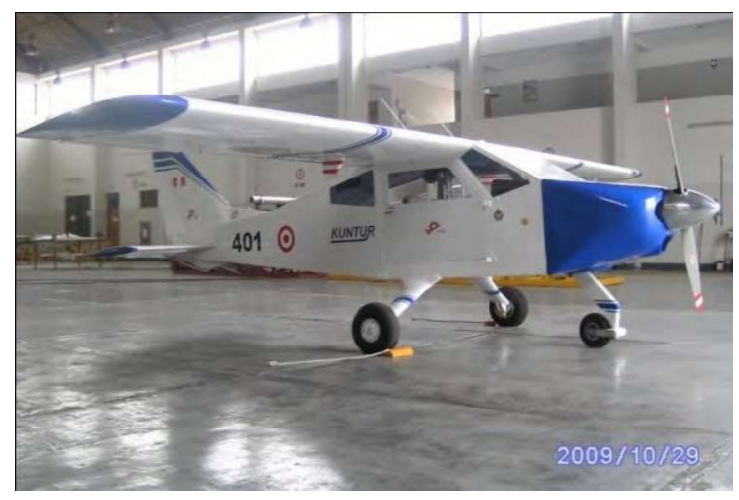

Figura 1: Aeronave de entrenamiento $B D-4 B P$ "KUNTUR"
El trabajo es realizado en dos dimensiones para lo cual se ha tenido que obtener un perfil del Kuntur y con el apoyo del software de simulación Fluent v6.2, se ha podido simular bajo diferentes condiciones hasta poder obtener los parámetros adecuados para nuestra investigación, esto es la velocidad, viscosidad, numero de Reynolds, tipo de flujo, tipo de orden del cálculo y residuales por interacción $[3,7,8]$.

Finalmente se obtuvieron los valores de los coeficientes de sustentación y arrastre los cuales se presentan en una tabla sencilla para lo cual se programo que los valores sean reportados luego de 5 interacciones y son colocados en Excel para poder obtener los valor promedios según el ángulo de ataque luego de ello, los valores son colocados en tablas para posteriormente ser graficados. Manifestándose la proporcionalidad de la fuerza con el coeficiente de sustentación y de arrastre, presentándose finalmente las conclusiones y la bibliografía utilizada.

\section{MATERIALES Y MÉTODOS}

Los materiales utilizados en este trabajo se manifiestan de la siguiente manera

Equipos:

- 01 aeronave BD-4BP (velocidad promedia de vuelo $77.778 \mathrm{~m} / \mathrm{s}$ )

- Software de simulación Fluent v6.2.16

- Software de mallado Gambit v2.2.30

- Computadora personal (procesador Pentium IV, capacidad física $120 \mathrm{~GB}, 1 \mathrm{~GB}$ de Ram, tarjeta de Video de $512 \mathrm{MB}$ ).

- Ploteador de imágenes.

- Material de escritorio

Instalaciones:

- Hangar de mantenimiento 9001 - SEMAN

- Laboratorio de Informática de la EODA - EOFAP

El método utilizado es el inductivo - deductivo; inductivo debido a que se realiza una revisión de los parámetros necesarios para aplicar las ecuaciones de sustentación-arrastre y a su vez utilizamos método deductivo por que con los datos obtenidos se podrá describir el comportamiento del Kuntur en aire a condiciones ambientales [5].

El Gambit no utiliza una metodología especial dado que simplemente es un software en el cual se realiza la construcción Geométrica y realiza una simulación para la determinación del número de geometrías, caras y zonas ya sea cuadrática o triangular, así también se fijan las condiciones de 
contorno, posteriormente se exporta en forma de archivo con extensión mesh.

El Fluent tienes varios métodos de cálculo, pero para nuestra investigación se ha utilizado el método de volúmenes finitos, con el cual se pueden realizar cálculos, que se asemejen a los valores reales $e$ inicialmente se realizan las interacciones bajo regimenes con flujo ideal $y$ posteriormente laminar no viscoso $[7,8]$.

\section{Descripción teórica}

La fuerza de sustentación es proporcional al coeficiente de sustentación del kuntur, la densidad del aire, el cuadrado de la velocidad de crucero de referencia a la cual llega el Kungur en vuelo despejado, y el área de referencia del Kuntur; con el apoyo de la bibliografía se puede despejar y definir el coeficiente de sustentación de la manera siguiente $[3,6,10]$ :

$$
C_{S}=\frac{F_{S}}{\frac{1}{2} \cdot \rho_{r e f} \cdot V_{r e f}^{2} \cdot A_{r e f}}
$$

Donde:

$C_{S}$ : Coeficiente de sustentación

$\mathrm{F}_{\mathrm{s}}$ : Fuerza de sustentación [N]

$\rho_{\text {ref }}:$ Densidad $[\mathrm{Kg} / \mathrm{m} 3]$

$V_{\text {ref: }}$ Velocidad del aire $[\mathrm{m} / \mathrm{s}]$

$A_{\text {ref: Área de la superficie alar }\left[\mathrm{m}^{2}\right]}$

Asimismo la fuerza de arrastre es proporcional al coeficiente de arrastre del kuntur, la densidad del aire, el cuadrado de la velocidad de crucero de referencia a la cual llega el Kuntur en vuelo despejado, y el área de referencia del Kuntur; definiendo el coeficiente de arrastre de la manera siguiente $[3,6,10]$ :

$$
C_{A}=\frac{F_{A}}{\frac{1}{2} \cdot \rho_{r e f} \cdot V_{r e f}^{2} \cdot A_{r e f}}
$$

Donde:

$C_{A}$ : Coeficiente de sustentación

$\mathrm{F}_{\mathrm{A}}$ : Fuerza de sustentación [N]

$\rho_{\text {ref }}:$ Densidad [Kg/m3]

$V_{\text {ref: }}$ Velocidad del aire $[\mathrm{m} / \mathrm{s}]$

$A_{\text {ref: }}$ Área de la superficie alar $\left[\mathrm{m}^{2}\right]$

\section{Descripción Experimental}

Para realizar el análisis numérico del perfil Kungur, primero se realizó el mallado, empleando el software GAMBIT v2.2.30. El Mallado final se muestra en la figura $\mathrm{N}^{\circ} 2$ y la figura $\mathrm{N}^{\circ}$.3. Se puede observar que el mallado es más fino cerca de la superficie delantera del perfil, debido a que es en esas zonas donde se producirá los mayores gradientes, aunque se debería mejorar el mallado en la parte posteroinferior, pero debido al tiempo de simulación se dejó del modo que se presenta.

En total el mallado consta de grillas, materiales, interfases, dominios y zonas, las cuales se detallan a continuación:

96029 nodos.

535 caras de la zona pared del Kuntur.

200 caras de la zona de simetría superior.

200 caras de la zona de simetría inferior.

200 caras de la zona final.

200 caras de la zona de ingreso.

190723 celdas triangulares.

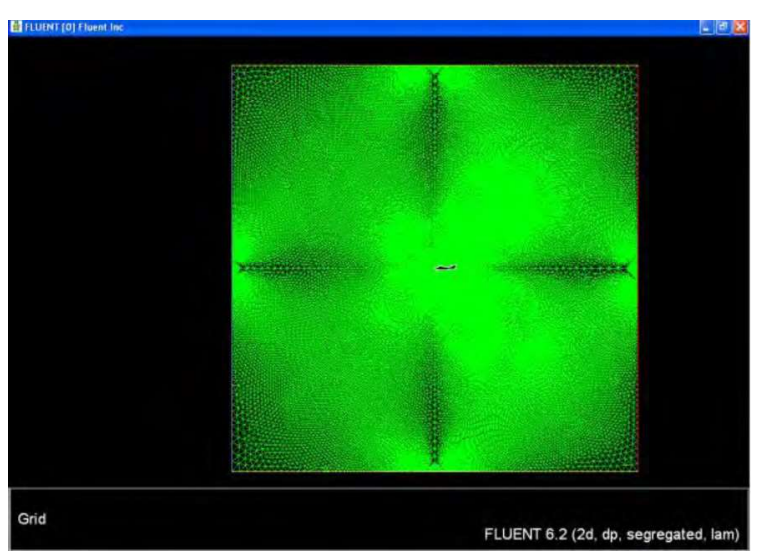

Figura 2: Mallado para perfil Kungur (escala aproximada 1:20)

Las condiciones de frontera establecidas son de entrada de velocidad, pared de simetría superior, pared de simetría inferior y salida de presión.

Paso seguido se procedió a establecer las condiciones de trabajo en el Fluent v6.2, empleando el segregated solver (2ddp, [4]), flujo estacionario, formulación implícita y un flujo ideal (no viscoso).

Para determinar el ángulo de incidencia del flujo de aire se determinó en las condiciones de frontera (boundary conditions) de entrada de velocidad (velocity inlet), la magnitud de $77.778 \mathrm{~m} / \mathrm{s}$ y las direcciones de acuerdo a los componentes de los vectores unitarios $\mathrm{X}, \mathrm{Y}$ : 
Componente $X=$ Cos $A O A^{\prime}$ y Componente $Y=$ Sen $A O A^{\prime}$ Donde: $A_{O A}$ : ángulo de ataque $\left(^{\circ}\right)$

Para el acoplamiento presión-velocidad se empleo el algoritmo SIMPLE [4], los factores de sub relajación fueron:

Presión

Densidad

Fuerzas en el Cuerpo:

Momentos

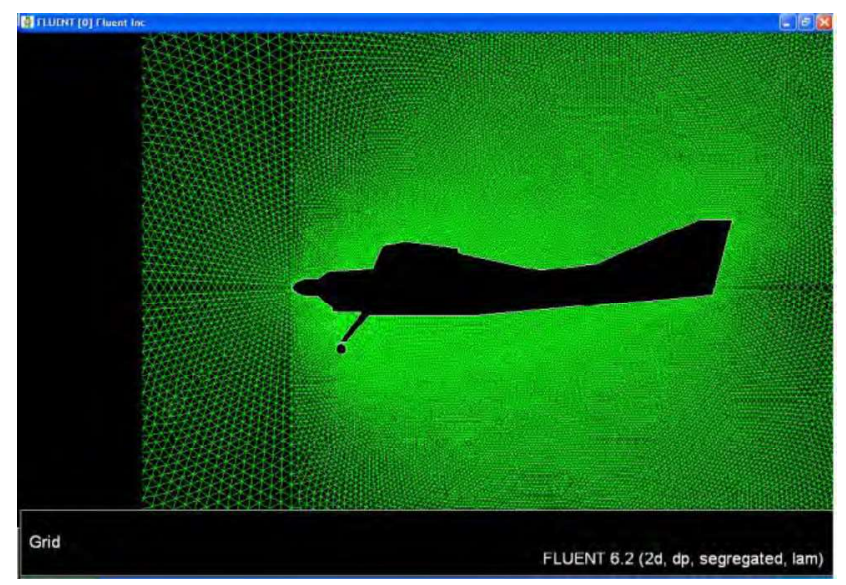

Figura 3: Mallado para perfil Kuntur (amplificado)

Los métodos de discretización fueron: Presión: PRESTO!

Para los momentos, primero se usó el método de First Order Upwind hasta obtener convergencia de los resultados para luego pasar al algoritmo más preciso de Second Order Upwind [4].

Los residuales fueron escalados y puestos bajo observación para obtener la convergencia de los mismos, estableciendo como criterio de convergencia residuales del orden de $10^{-6}$.

Se procedió a las iteraciones para cada ángulo de ataque hasta obtener convergencia en los resultados.

Tabla I: Condiciones establecidas para la obtención de coeficiente de Sustentación y arrastre

\begin{tabular}{|c|c|}
\hline \multicolumn{1}{|c|}{ Parámetros } & Valores \\
\hline$\rho:$ Densidad $[\mathrm{Kg} / \mathrm{m} 3]$ & 1,225 \\
\hline $\begin{array}{l}\mathrm{V}_{\text {ref: }} \text { Velocidad de referencia del } \\
\text { Kuntur o velocidad de simulación } \\
\text { del aire [m/s] }\end{array}$ & 77.778 \\
\hline
\end{tabular}

\begin{tabular}{|l|l|}
\hline & \\
\hline $\begin{array}{l}\text { S ref: Área de referencia superficie } \\
\text { del kuntur }\left[\mathrm{m}^{2}\right]\end{array}$ & 1 \\
\hline
\end{tabular}

Los valores obtenidos fueron evaluados y se tomó una muestra a la cual se le aplicó la estadística respectiva. Con ayuda de la fuerza de sustentación y arrastre obtenida y la ecuación $\mathrm{N}^{0} 1$ y $\mathrm{N}^{0} 2$, se obtuvo el coeficiente de sustentación y arrastre en función del ángulo de ataque, el cual se muestra en la gráfica $\mathrm{N}^{\circ} 1$.

\section{RESULTADOS Y DISCUSIÓN}

Los resultados obtenidos con el FLUENT 6.2 se lograron después de aproximadamente 1 hora de iteraciones para cada ángulo de ataque. Los resultados que se presentan fueron obtenidos simulando un fluido ideal no viscoso, situación que no se adapta a la realidad pero que sin embargo nos permite tener resultados muy cercanos para el caso del coeficiente de sustentación (gráfica $\mathrm{N}^{0} 1$ ) y coeficiente de arrastre (gráfica $\mathrm{N}^{\circ} 2$ ).

Como se puede apreciar en la grafica $\mathrm{N}^{0} 1$, el coeficiente de sustentación aumenta a medida el ángulo de ataque con valores que oscilan entre 0,5 y 2,0 para ángulos entre 0 y 24 grados, presenta una ascendencia hasta el ángulo de $18^{\circ}$ a partir del cual los valores del coeficiente de sustentación empiezan a descender.

Así mismo en la gráfica $\mathrm{N}^{\circ} 2$, el coeficiente de arrastre aumenta a medida que el ángulo de arrastre aumenta sin presentar variaciones abruptas.

Como sabemos el coeficiente de sustentación puede ser positivo o negativo, mientras el coeficiente de arrastre es siempre positivo ${ }^{1}$. Dicho de otra manera el coeficiente de sustentación está asociado con la factibilidad de que la aeronave pueda ascender (coeficiente de sustentación con pendiente positiva) o descender (coeficiente de sustentación con pendiente negativa); mientras que el coeficiente de arrastre es positivo por que siempre existirá una fuerza que oponga al movimiento no obstante que la velocidad o propulsión de la aeronave aumente o disminuya.

\footnotetext{
${ }^{1}$ El análisis sobre los coeficientes aerodinámicos son desarrollados en detalle por I. Carmona, en el cap. 4 del libro Aerodinámica y actuaciones del avión.
} 


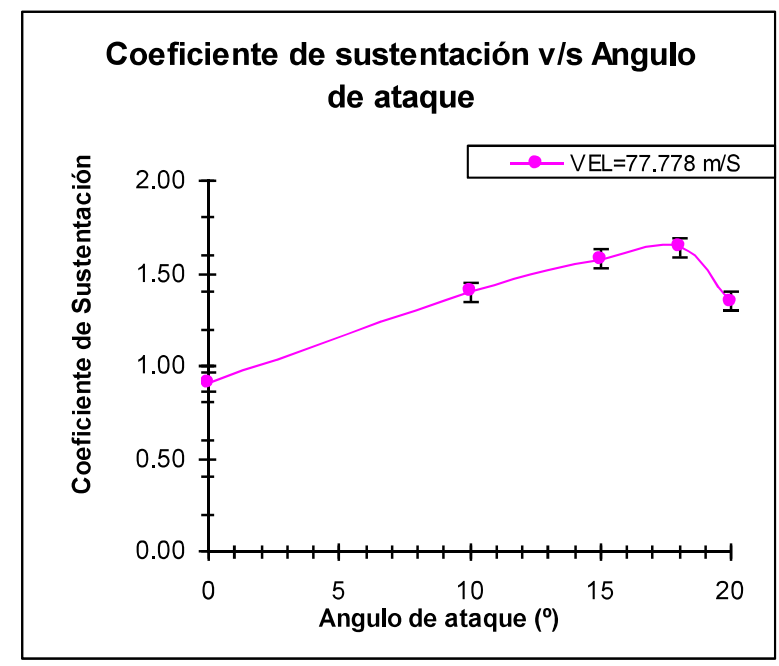

Gráfica 1: Datos Simulados con flujo ideal del Coeficiente de Sustentación V/S Angulo de Ataque con velocidad de $77.778 \mathrm{~m} / \mathrm{s}$.

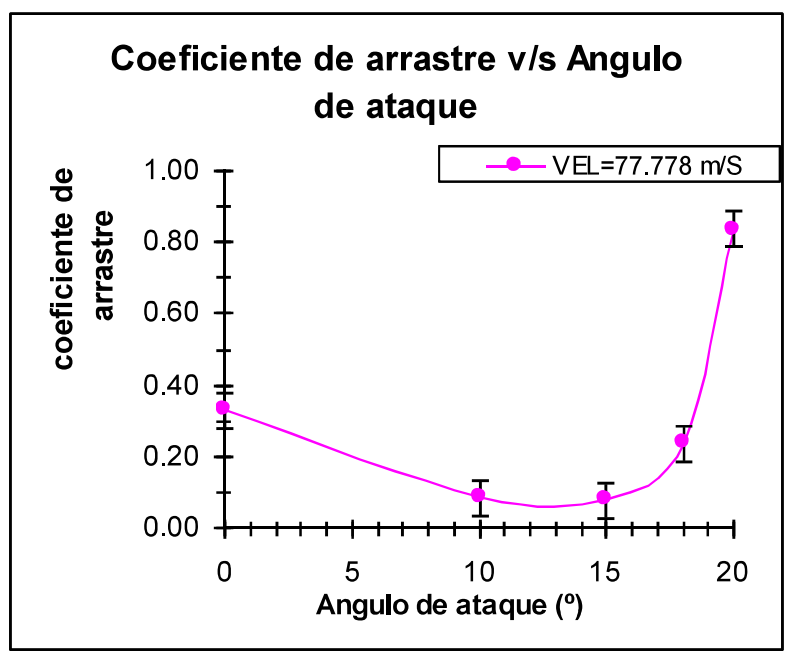

Gráfica 2: Datos simulados con flujo ideal del Coeficiente de arrastre V/S Angulo de Ataque con velocidad de $77.778 \mathrm{~m} / \mathrm{s}$.

Los valores de velocidad oscilan mucho en las zonas de resistencia como son la parte cercana al tren de aterrizaje delantero y a la parte superior del a la principal (ver figura $\mathrm{N}^{\circ} 4$ ); por dicho motivo se puede apreciar que hay un aumento de los valores de velocidad en el tren de aterrizaje que es superior con respecto a cualquier punto de la aeronave; así mismo, existe una relación entre la presión y la velocidad de la aeronave las cuales están inversamente ligadas, por lo que podemos asegurar que el tren de aterrizaje será el punto con menor presión [8].

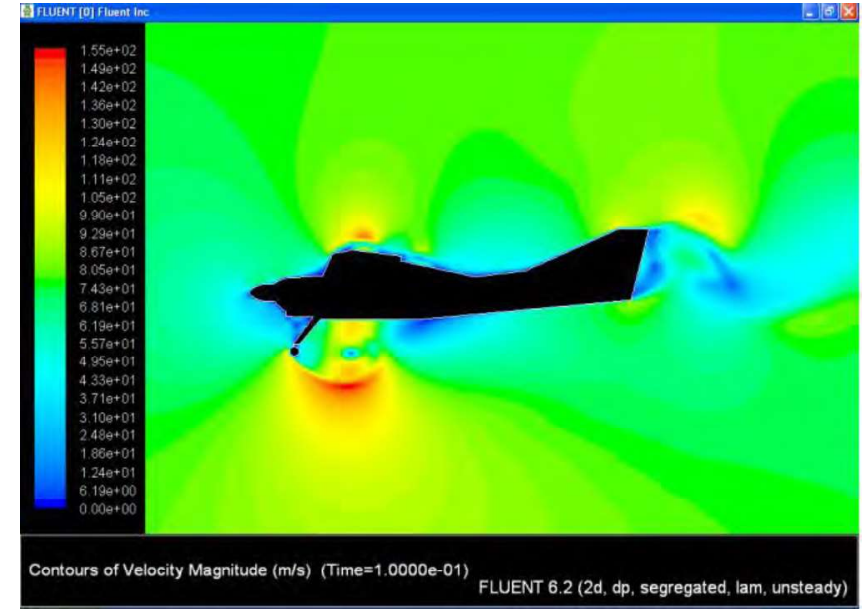

Figura 4: Presentación del contorno de velocidades de la Simulación del perfil del Kungur con el CFD Fluent V6.2 para ángulo de ataque de $0^{\circ}$ y velocidad de $77.778 \mathrm{~m} / \mathrm{s}$.

\section{CONCLUSIONES}

Se concluye que cuando el ángulo de ataque supera los $18^{\circ}$, se observa una abrupta variación de la fuerza de sustentación lo que originaría que la aeronave se precipite a tierra, es decir la aeronave entra en perdida.

De la figura $\mathrm{N}^{\circ} 4$ se concluye que los valores máximos de velocidad serán de $155 \mathrm{~m} / \mathrm{s}$ en tren de aterrizaje y $43 \mathrm{~m} / \mathrm{s}$ en la parte superior del ala principal.

\section{AGRADECIMIENTOS}

El agradecimiento especial por su apoyo y facilidades con la aeronave Kuntur al COR. FAP Pedro Estremadoyro Cabrera; así también a las autoridades de la EOFAP al MAG FAP Rodolfo García Esquérre y al Com. FAP Miguel Núñez Talledo.

\section{REFERENCIAS}

[1] Servicio de mantenimiento de la FAP SEMAN. Manual de ingeniería del avión BD4BP. Perú. 2009.

[2] Servicio de mantenimiento de la FAP SEMAN. Manual de fabricación del avión BD4BP. Perú. 2009.

[3] J. H. Ferzirger and M. Peric. Computational methods for fluid dynamic. 3 rd Edition. Springer. Germany. 2002.

[4] Fluent Inc. FLUENT 6.2: Tutorial guide. USA. 2006.

[5] Day, Robert A. Cómo escribir y publicar trabajos de investigación. 3era Ed. OPS. 
Viena. 2005.

[6] Bertin John J. and Smith Michael L. Aerodynamics for engineers. USA. 1979.

[7] Zienkiewics, O.C.; Taylor, R. L. The Finite Element Method. Volume 3: Fluid Dynamics. Fifth Edition. Ed. Butterworth, 2000.

[8] Ferziger, J. H.; Peric, M. Computational Methods for Fluid Dynamics. Third Edition. Ed. Springer, 2002.

[9] Dole, Charles E. Teoría de vuelo y aerodinámica. España. 1984.

[10] Carmona, Isidoro. Aerodinámica y actuaciones del avión. 5ta Ed. Editorial Paraninfo. Madrid. 1983.

E-mail: lunajosern@hotmail.com 